\title{
Dynamics of a Random Laser above Threshold
}

\author{
Gijs van Soest,* Frank J. Poelwijk, Rudolf Sprik, and Ad Lagendijk \\ Van der Waals-Zeeman Instituut, Universiteit van Amsterdam, Valckenierstraat 65, 1018 XE, Amsterdam, The Netherlands
}

(Received 5 July 2000)

\begin{abstract}
We have performed enhanced backscattering experiments in a high gain random laser, under circumstances where a stationary theory predicts the laser intensity to diverge. Above the laser threshold the observed backscatter cone changes only gradually, not showing any signs of the divergence. We present measurements and theory - generalized laser equations with a diffusive transport term for pump and laser light - to explain the observed behavior. The population dynamics prevent an indefinite growth of the intensity. Time dependence is essential for a theory of random lasers above the laser threshold.
\end{abstract}

DOI: $10.1103 /$ PhysRevLett.86.1522

Random lasers are strongly scattering media with optical gain. These systems have many features in common with more conventional lasers based on an optical gain medium enclosed in a cavity with two mirrors to enhance stimulated emission. For example, a threshold for lasing action and frequency narrowing have been observed in random lasers based on multiply scattering colloidal dispersions in dyes $[1,2]$. Evidently, the optical properties of random lasers are quite different from conventional lasers: the propagation of pump and fluorescence light is diffusive, and - in the absence of well-defined cavity modes - there is no "preferred direction" in feedback and loss processes. The ambiguity as to what exactly constitutes the loss in a random laser, how optical feedback works if it is nondirectional, and the theoretical prediction of an intensity divergence, have led to a continuing debate about what happens at, and above, the laser threshold.

The random laser has been likened to neutron transport in fissionable material. Both systems are described by diffusive transport with amplification. Transport through the interfaces of the system is a loss factor. If the volume in which this amplification occurs is so large that the transport cannot fully compensate the gain, the intensity diverges. In an atomic bomb this occurs at the critical mass, and the result is a nuclear explosion. Letokhov predicted a similar "explosion" [3] for multiple scattering of light in an amplifying medium at a critical volume. Several researchers have subsequently identified [4,5] this explosion with the random laser threshold. The divergence of the intensity has hampered theoretical efforts above the laser threshold.

In this Letter we demonstrate that in a realistic system the explosion does not exist, and consequently is not the same as a laser threshold, which does exist. At the laser threshold the population inversion is fixed at a level where the (local) gain exactly compensates the (local) loss, such as in an ordinary laser the modal gain equals the modal loss. A description of the system that allows for the intrinsic dynamics associated with the threshold crossing does not predict the divergence. We include $\beta$, the fraction of spontaneous emission contributing to the laser process, and
PACS numbers: 42.55.-f, 42.25.Dd, 42.65.Sf, 78.45.+h

establish the validity of the model by a successful comparison with measurements.

We experimentally probed the gain dynamics in a random laser by enhanced backscattering. Enhanced backscattering (EBS) has evolved from being a subject of study in itself [6] into a tool that can be used to study transport of waves in random media in a very precise and quantitative manner [7]. The shape of the backscatter cone is determined by the transport distance of the light in the medium. The intensity in an amplifying medium grows exponentially with path length $\Lambda, e^{\kappa_{g} \Lambda}$, where $\kappa_{g}$ is the gain coefficient. Consequently, gain in a multiplescattering medium results in an enhancement of long light paths compared to the passive material. The long light paths constitute the top of the EBS cone: a relatively larger contribution of long paths yields a sharper and narrower cone $[5,8]$. This sensitivity to long paths makes EBS particularly well suited for testing the divergence behavior.

Our measurements of EBS in high gain amplifying random media were performed with samples consisting of $220 \mathrm{~nm}$ diameter $\mathrm{TiO}_{2}$ colloidal particles suspended in $1.0 \mathrm{mM}$ sulforhodamine B laser dye in methanol. The samples are contained in a plastic cell with a glass front window. The cell slowly spins to prevent sedimentation and to assist speckle averaging.

The dye $/ \mathrm{TiO}_{2}$ suspension is optically pumped with a frequency doubled Nd:YAG laser (Coherent Infinity, wavelength $532 \mathrm{~nm}$, pulse duration $2 \mathrm{~ns}$ ). The pump fluence range at the sample position is $0-140 \mu \mathrm{J} / \mathrm{mm}^{2}$. We use a low energy $\left(3 \mu \mathrm{J} / \mathrm{mm}^{2}\right)$ probe pulse from an optical parametric oscillator (Coherent XPO) tuned to the maximum of the fluorescence band of the dye $(590 \mathrm{~nm}$, pulse duration $4.4 \mathrm{~ns}$ ) for EBS. The pump and probe beams ( $3 \mathrm{~mm}$ diameter, $20 \mathrm{~Hz}$ pulse rate) coincide in space and time. The probe beam enters via a beam splitter to allow intensity measurements in the exact backscattering direction.

The scattered light is collected through an interference filter and a focusing lens on a CCD camera to record the EBS cone. We accumulate 51 to 204 different speckles in each exposure, depending on the collected intensity. The 
angular resolution is $1 \mathrm{mrad}$ (limited by the probe beam divergence).

Earlier EBS experiments [8,9] were performed with materials in which the laser threshold could not be reached. In our sample the laser threshold is found to be at a pump fluence of $10 \mu \mathrm{J} / \mathrm{mm}^{2}$ from the width of the fluorescence spectrum as a function of the pump pulse energy. In Fig. 1 some examples of EBS cones are given for different pump energies: below, near, and above threshold. From the backscattering cone obtained without pump we infer that the transport mean-free path $\ell=3 \mu \mathrm{m}$.

Both the full width at half maximum (FWHM) and enhancement factor $(E)$ become smaller with increasing pump energy. The enhancement factor is the ratio between the intensity at the peak of the cone and the background at exact backscattering, and has a (gain-independent) value of maximally 2 that is diminished by angle-independent contributions to the intensity. The FWHM is related to the transport length (for a cone without gain it is $\alpha \ell^{-1}$ ) and provides a measure to compare the cones at varying pump energy. Figure 2 shows the FWHM of the EBS cones as a function of pump fluence. The larger fraction of long light paths at high gain reduces the width of the EBS cone, but there is no sign of a divergence [5] or a sudden change in behavior at the threshold crossing. The decrease of $E$ from 1.65 to 1.25 is due to the incoherent fluorescence component in the collected light, which becomes stronger for higher pump energies.

We introduce a model for the inversion and laser light dynamics in space and time, the results of which are tested by a comparison with the EBS experiment. EBS is sensitive to the exact gain profile, $\kappa_{g}(z)[10]$. Since the in-

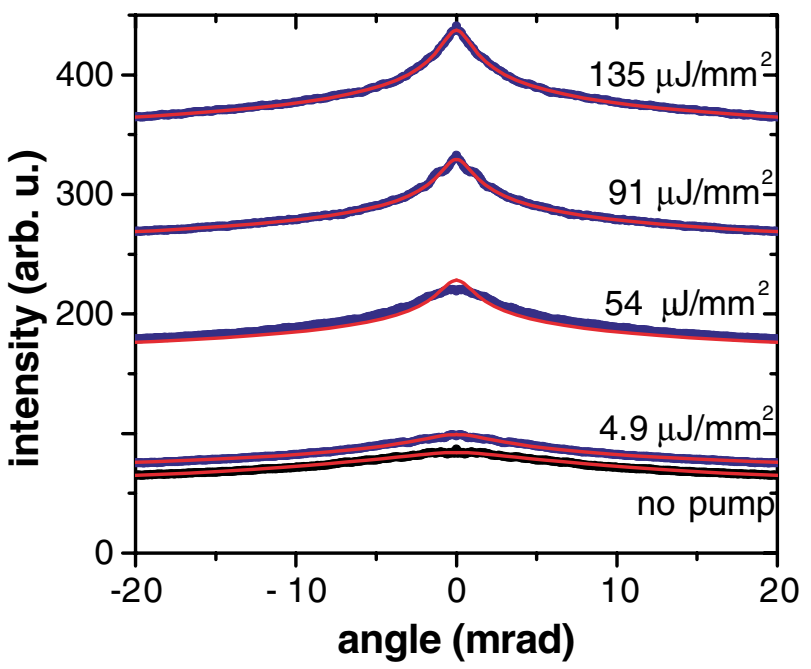

FIG. 1 (color). Points: enhanced backscattering cones for pump fluences ranging from 0 to $135 \mu \mathrm{J} / \mathrm{mm}^{2}$. Lines: obtained from a time-dependent description of the system response to a pump pulse. The experimental results are accurately reproduced by the theory, except for intermediate pump energies (approximately $20-70 \mu \mathrm{J} / \mathrm{mm}^{2}$; one example shown) where the relaxation oscillations dominate the temporal inversion profile. elastic length $\ell_{a}$ of the neat dye solution is $110 \mu \mathrm{m}$ at the pump wavelength, the diffuse absorption length $L_{a}=$ $\sqrt{\ell \ell_{a} / 3}=10.5 \mu \mathrm{m}$. Even if the absorption is strongly saturated, the amplification mainly occurs in a layer with a thickness $<10 \ell$.

The gain distribution is calculated with a set of spaceand time-dependent generalized laser equations, incorporating the multiple-scattering character of the transport by the inclusion of a diffusion term $\propto D \nabla^{2} W$, which maps on the cavity loss term in the description of a conventional laser [11]. We use a slab geometry and plane wave illumination, leaving only one relevant spatial coordinate. Spontaneous emission is the seed of the laser light.

The generalized time-dependent random laser equations are

$$
\begin{aligned}
\frac{\partial W_{\ell}}{\partial t} & =D \nabla_{z}^{2} W_{\ell}+\left(\sigma_{e} c n_{1}-\sigma_{r} c n_{0}\right) W_{\ell}+\frac{\beta}{\tau} n_{1}, \\
\frac{\partial W_{p}}{\partial t} & =D \nabla_{z}^{2} W_{p}-\sigma_{a} c n_{0} W_{p}+\frac{1}{\ell} I_{\text {in }}, \\
\frac{\partial n_{1}}{\partial t} & =\sigma_{a} c n_{0} W_{p}-\left(\sigma_{e} c n_{1}-\sigma_{r} c n_{0}\right) W_{\ell}-\frac{1}{\tau} n_{1} .
\end{aligned}
$$

Here, $W_{\ell, p}(z, t)$ is the luminescence and pump light density, $n_{0,1}(z, t)$ is the density of dye molecules in the ground and excited states, with $n=n_{0}+n_{1}$ the total molecular density, $c$ is the speed of light in the medium, $\sigma_{a, e, r}$ are the molecular absorption, stimulated emission and reabsorption cross sections, and $D=\frac{1}{3} c \ell$ is the diffusion coefficient for light. Further, $\tau$ is the excited state lifetime, and $I_{\text {in }}(z, t)$ is the incident pump flux.

$\beta$ is the fraction of spontaneous emission that may seed the laser process, and is determined by the overlap in wave-vector space between the spontaneous emission

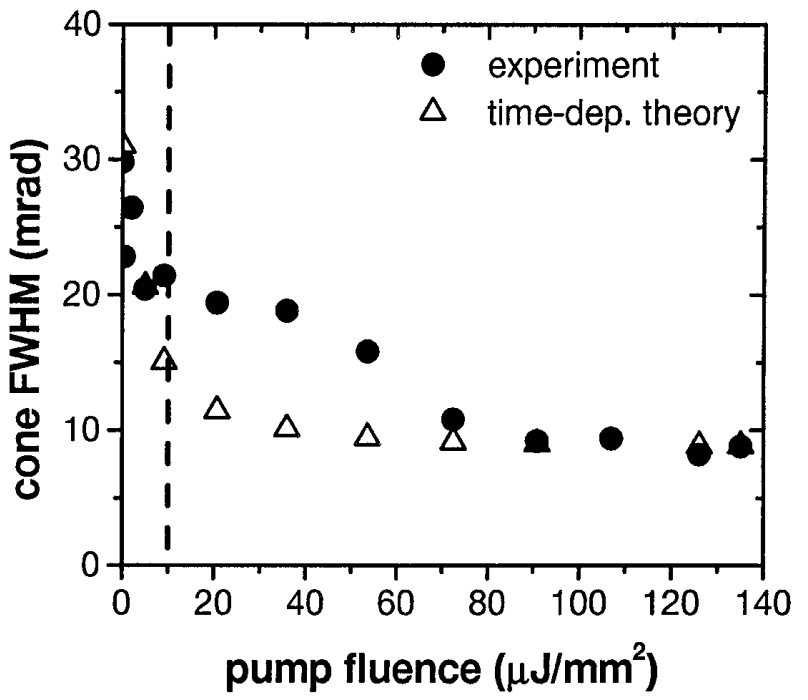

FIG. 2. The full widths at half maximum of the backscatter cones as a function of pump fluence. The dashed line indicates the threshold, obtained from an independent measurement. 
and the laser field. Its significance in the context of random lasers was not recognized earlier. In conventional lasers, $\beta$ is small $\left(\sim 10^{-8}-10^{-5}\right)$ because of the small solid angle spanned by the cavity modes. As a result of the nondirectional feedback in a random laser, the spatial distinction between lasing and nonlasing modes vanishes and the only criterion is the spectral overlap of emission spectra below and above threshold. This method leads to an estimate of $\beta=0.1$, signifying that $10 \%$ of the modes of the system participate in lasing. In our calculation, $\beta$ serves to take into account the spectral narrowing without having to calculate the full spectral dependence of the system.

This system of coupled nonlinear partial differential equations is solved by the method of lines [12]. The input for the calculation is the material parameters, which are all (except for the quantum efficiency [13]) experimentally determined by us, and the pump pulse incident on the medium. The calculation is carried out for a sample of $200 \ell=600 \mu \mathrm{m}$ thickness, over a time span of $12 \mathrm{~ns}$. The results are $W_{\ell}(z, t), W_{p}(z, t)$, and $n_{1}(z, t) / n$; some examples are presented in Figs. 3 and 4. The gain coefficient is $\kappa_{g}(z, t)=n_{1}(z, t) \sigma_{e}=240 \frac{n_{1}}{n}(z, t) \mathrm{cm}^{-1}$.

We now discuss the key features of the spatiotemporal inversion $\left(n_{1}\right)$ and luminescence density $\left(W_{\ell}\right)$ profiles. The most important observation is that for any pump rate a finite equilibrium value for the luminescence density exists. Before reaching that value, the system may go through a relaxation oscillation in which the $W_{\ell}$ and $n_{1}$ can become larger than the equilibrium level, but only for a short time. The saturation of the gain at high luminescence density (deexcitation of the gain medium by stimulated emission) restores the equilibrium, preventing an indefinite growth of $W_{\ell}$. This relaxation oscillation acts as a safety valve in random lasers. In the stationary description, the relax-

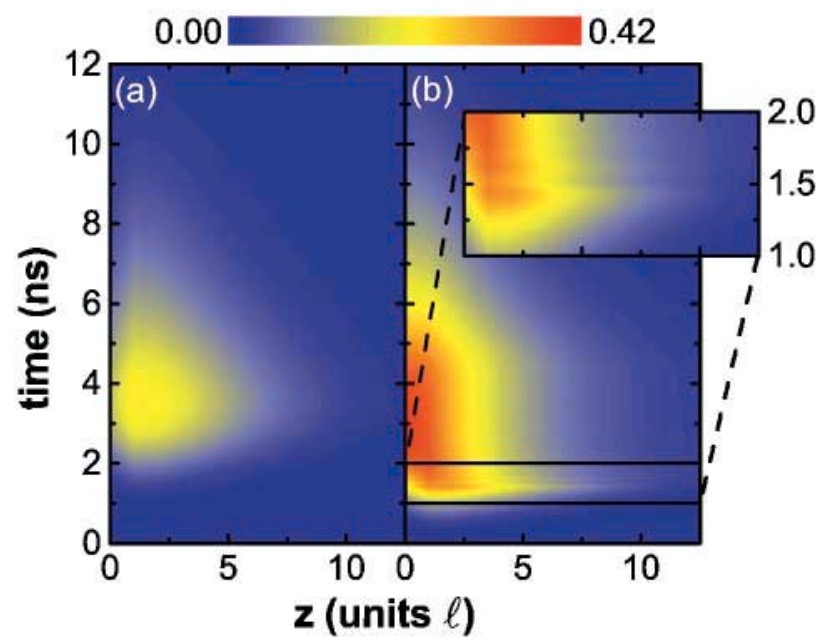

FIG. 3 (color). Calculated spatiotemporal inversion $n_{1} / n$ for pump fluences of (a) $4.9 \mu \mathrm{J} / \mathrm{mm}^{2}$ and (b) $53.7 \mu \mathrm{J} / \mathrm{mm}^{2}$. The inset shows a close-up of the oscillation that occurs above threshold. ation oscillation is not allowed and an explosion cannot be circumvented.

Only a relatively thin $(<10 \ell)$ layer with significant inversion, and, consequently, a high gain coefficient, develops at the arrival of the pump pulse. Larger $z$ 's are not reached by the pump light, which either gets absorbed or is scattered out of the medium. Because the luminescence has a much lower absorption, $W_{\ell}$ does extend through the entire system.

The light and population dynamics are much like those of conventional lasers. For a pump energy below threshold [Fig. 3(a)], $n_{1}$ follows the pump intensity. $W_{\ell}(t)$ is practically zero for low pump rates; at the crossing of the pump threshold [Fig. 3(b), inset] it shoots through its equilibrium value and starts a decaying oscillation. After the oscillation, $W_{\ell}$ is proportional to the pump, and $n_{1}$ is clamped at the threshold value, which is set by the loss and hence is essentially independent of pump energy. When the pump falls below the threshold level, $W_{\ell}$ drops and $n_{1}$ starts to decay spontaneously $[t>6$ ns in Fig. 4(b)].

The dynamics occur simultaneously throughout the system (see Fig. 3), indicating that the transport is fast compared to other processes; in the analogy with a normal laser, the cavity decay time $\tau_{c}$ is short. This condition indeed gives rise to spiking. $\tau_{c}$ in a random laser is derived from the diffuse transport time over a relevant length $L_{x}$, e.g., the thickness of the amplifying layer, $\tau_{c}=L_{x}^{2} / D$. Using $L_{x}=5 \ell$ yields $\tau_{c} \approx 1 \mathrm{ps}$. In a conventional laser, the relaxation oscillation frequency $\omega_{r} \approx \sqrt{(r-1) /\left(\tau \tau_{c}\right)}$ and decay rate $\gamma_{r} \approx r /(2 \tau)$ [11], where $r$ is the pump rate, normalized at the threshold value. The random laser behaves similarly: just above threshold the oscillations are slower and longer lived than for very high pump energies. We typically find oscillation times of the order of $100 \mathrm{ps}$,

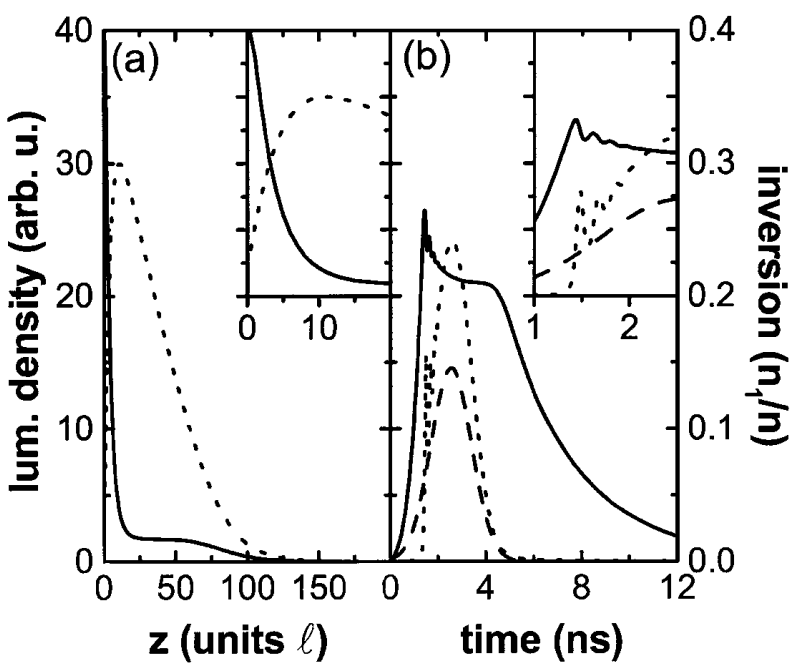

FIG. 4. Space and time cross sections through the data of Fig. 3(b). (a) $n_{1}(z)$ (solid line) and $W_{\ell}(z)$ (dotted line) at $t=3 \mathrm{~ns}$. The important range of small $z$ 's is shown in the inset. (b) $n_{1}(t)$ and $W_{\ell}(t)$ at $z=3 \ell$ and the incident pulse (dashed line). The inset displays the relaxation oscillation. 
lasting for $1 \mathrm{~ns}$, values that agree with the estimated diffusion $\tau_{c}$. These results qualitatively confirm the Monte Carlo simulations by Berger et al. [14] for short pulses and thin slabs.

The red lines in Fig. 1 are obtained [10] from the $n_{1}(z, t)$ found with this model: the agreement between experimental data and theoretical description is excellent for low and high pump energies. Initially, the width of the EBS cone drops quickly with increasing pump pulse energy. Far above threshold, the FWHM saturates (at $\approx 10 \mathrm{mrad}$, depending on system parameters) due to the pump independence of the above threshold $n_{1}(z)$. In the analysis of $n_{1}(z)$, the time variation is a subtle issue. Since the EBS process itself samples the medium on the time scale needed to build up a cone, it only senses slow variations in $n_{1}(z)$. The longest paths that contribute in an experiment with an angular resolution of $1 \mathrm{mrad}$ have a separation between entrance and exit points of $d=10^{3} \lambda \approx$ $600 \mu \mathrm{m}$. The diffusive transport time over this distance is $d^{2} / D \approx 1.7 \mathrm{~ns}$. We mimic this property by low-pass filtering the data, and subsequently averaging $n_{1}(z, t)$ in time windows of length $d^{2} / D$. The cones that result from these mean inversion profiles are summed, each weighted with the mean probe intensity in its window. This procedure largely overcomes the dominance of the nearly critical $\kappa_{g}(z)$ occurring in the relaxation oscillation: long paths, needed for the divergence to happen, do not have time to build up in the $\approx 50 \mathrm{ps}$ that the high inversion lasts. This demonstrates once more that the dynamic picture, although allowing for large inversion densities, prevents the explosion. We stress that a theory that does not incorporate the full dynamics fails to reproduce the cones completely. In particular a static approach predicts an extremely small width and diverging height when using the same parameters.

For pump fluences between 20 and $70 \mu \mathrm{J} / \mathrm{mm}^{2}$ the theory deviates from the experimental results (Figs. 1 and 2 ). This discrepancy is due to the entanglement of the time scales of transport and variation of $n_{1}(z)$. Since $n_{1}(z)$ changes faster than the time needed for the formation of a backscatter cone, the reversibility of transport in the medium is affected. A wave traversing the medium along a certain path experiences a spatiotemporal gain profile that is, in principle, different from the profile seen by the wave in the reversed path. This reduces the interference contrast in the scattered light, as the two waves no longer have equal amplitudes when exiting the medium. This imbalance is especially prominent just above threshold, where the long-lived oscillations make up an important part of the temporal gain profile. Long light paths are most strongly influenced by the changing $n_{1}(z)$. Their interference contribution is smaller than inferred from the averaged gain profile, and the measured EBS cone is broader than given by our theory. A simulation of dynamic EBS backs up this explanation, showing a cone broadening of the correct magnitude due to the inequality of interfering paths.

This work is part of the research program of the "Stichting voor Fundamenteel Onderzoek der Materie (FOM)," which is financially supported by the "Nederlandse Organisatie voor Wetenschappelijk Onderzoek (NWO)."

*Email address: vansoest@phys.uva.nl

[1] N. M. Lawandy, R. M. Balachandran, A. S. L. Gomes, and E. Sauvain, Nature (London) 368, 436 (1994).

[2] G. van Soest, M. Tomita, and A. Lagendijk, Opt. Lett. 24, 306 (1999).

[3] V.S. Letokhov, Sov. Phys. JETP 26, 835 (1968).

[4] See, e.g., C. W. J. Beenakker, J. C. J. Paasschens, and P. W. Brouwer, Phys. Rev. Lett. 76, 1368 (1996); A. A. Burkov and A. Yu. Zyuzin, Phys. Rev. B 55, 5736 (1997); P. Pradhan and N. Kumar, Phys. Rev. B 50, 9644 (1994); X. Jiang and C. M. Soukoulis, Phys. Rev. B 59, 6159 (1999); M. Patra and C. W. J. Beenakker, Phys. Rev. A 60, 4059 (1999).

[5] A. Yu. Zyuzin, Europhys. Lett. 26, 517 (1994).

[6] Y. Kuga and A. Ishimaru, J. Opt. Soc. Am. A 8, 831 (1984); M. P. van Albada and A. Lagendijk, Phys. Rev. Lett. 55, 2692 (1985); P. E. Wolf and G. Maret, Phys. Rev. Lett. 55, 2696 (1985).

[7] F. J. P. Schuurmans, M. Megens, D. Vanmaekelbergh, and A. Lagendijk, Phys. Rev. Lett. 83, 2183 (1999).

[8] D. S. Wiersma, M. P. van Albada, and A. Lagendijk, Phys. Rev. Lett. 75, 1739 (1995).

[9] P. C. de Oliveira, A. E. Perkins, and N. M. Lawandy, Opt. Lett. 21, 1685 (1996).

[10] W. Deng, D. S. Wiersma, and Z. Q. Zhang, Phys. Rev. B 56, 178 (1997).

[11] See, e.g., A.E. Siegman, Lasers (University Science Books, Mill Valley, 1986).

[12] IMSL routine DMOLCH, IMSL Math/Library Users Manual (IMSL Inc., 2500 CityWest Boulevard, Houston, Texas 77042).

[13] Kodak Laser Dyes (Eastman Kodak Company, Rochester, 1987); J. M. Drake, R. I. Morse, R. N. Steppel, and D. Young, Chem. Phys. Lett. 35, 181 (1975).

[14] G. A. Berger, M. Kempe, and A.Z. Genack, Phys. Rev. E 56, 6118 (1997). 